\section{Stabilization of Networked Control Systems With a Logic ZOH}

Junlin Xiong and James Lam, Senior Member, IEEE

\begin{abstract}
The technical note is concerned with the stabilization problem of networked control systems. A general framework is proposed firstly, where the zero-order hold has the logical capability of choosing the newest control input packet. The continuous-time process is discretized as a system with input delays. Then a sufficient condition for testing the stability of the discretized system and two sufficient conditions for designing a stabilizing controller are established based upon the Lyapunov theory. Finally numerical examples and simulations are used to illustrate the developed theory.
\end{abstract}

Index Terms-Networked control systems, packet losses, stabilization, time delays.

\section{INTRODUCTION}

Networked Control Systems (NCSs) have been an active research topic in recent years. They differ from traditional control systems in that the connections of their components are via shared communication networks instead of point-to-point wiring. The use of the shared communication networks between control system components is mainly motivated by lower cost, easier maintenance and higher reliability of the closed-loop systems [1]. The applications of NCSs can be found in many fields such as automobiles, aircrafts, and HVAC systems [1]. However, the introduction of the networks complicates the analysis and synthesis problems of control systems. Network-induced time delays, packet losses and signal quantization are major issues in front of any NCS designer (see [2]-[4] for a general introduction to NCSs).

Currently, several methodologies have been proposed to tackle the control problems of NCSs, and may be divided into the following three classes: a) Control system components are designed by traditional theory, much effort goes into the design and scheduling of the communication networks [1], [5]-[8]; b) The characteristic of the networks is given in advance, the main task is to design the control system components under those communication constraints [9]-[20]; c) The communication networks and the control system components are co-designed [21].

Generally, time delays and packet losses are two essential issues that need careful consideration in an NCS design. They can be handled separately [9]-[14] or simultaneously [15]-[20]. An appealing idea is modeling them as input delays so that the approaches developed for time-delay systems can be adapted [15]-[20]. In [15]-[17], the $H_{\infty}$ control problem was studied in the continuous-time domain, where the input delays belong to a given interval [16], [17] or are divided into two parts [15]. In [18], the input delays were assumed to be two homogeneous Markov chains. A necessary and sufficient condition was established for the stochastic stability of NCSs through augmentation technique. The authors of [19], [20] assumed that the input delays are

Manuscript received August 03, 2006; revised June 18, 2007 and May 01, 2008. Current version published February 11, 2009. This work was supported in part by RGC HKU 7031/07P. Recommended by Associate Editor C. Abdallah.

The authors are with the Department of Mechanical Engineering, University of Hong Kong, Hong Kong, China (e-mail: junlin.xiong@gmail.com; james. lam@hku.hk).

Color versions of one or more of the figures in this technical note are available online at http://ieeexplore.iee.org.

Digital Object Identifier 10.1109/TAC.2008.2008319

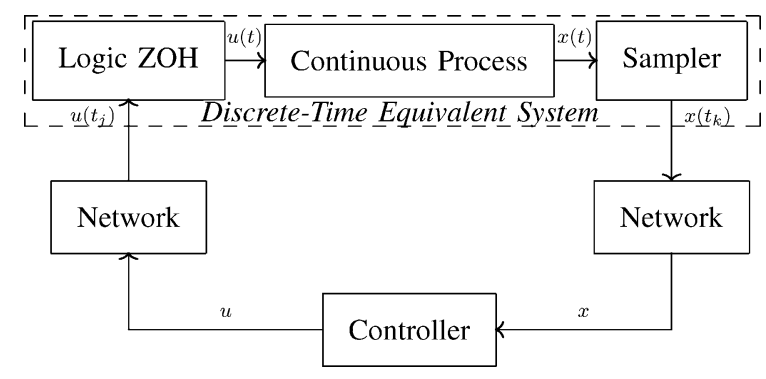

Fig. 1. General framework of networked control systems.

between two positive integers, and studied the stability problem of discrete-time NCSs.

In this technical note, the networks are taken as given conditions and the stabilization problem of NCSs is studied from the viewpoint of zero-order hold $(\mathrm{ZOH})$. The $\mathrm{ZOH}$ is assumed to be both time-driven and event-driven, and has the logical capability of comparing the time stamps of the arrived control input packets and choosing the newest one to control the process. Under such a configuration, the overall NCS is discretized as a linear discrete-time system with input delays. A sufficient condition for testing the stability of the discretized NCSs and two sufficient conditions for designing a networked controller are established such that the NCS operating in closed-loop is stable. The approach is based upon the Lyapunov theory, and the conditions are given in terms of linear matrix inequalities (LMIs). Moreover, several numerical examples and simulations are used to illustrate the efficiency of the developed theory.

Notation: $\mathbb{Z}_{+}$is the set of nonnegative integers. $\mathbb{R}^{n}, \mathbb{R}^{m \times n}$ and $\mathbb{S}^{+}$denote, respectively, the $n$-dimensional Euclidean space, the set of $m \times n$ real matrices and the set of $n \times n$ real symmetric positive definite matrices. Notation $X<0$ means that $-X \in \mathbb{S}^{+} . I$ is the identity matrix of compatible dimensions. The superscript " $T$ " denotes the transpose for vectors or matrices.

\section{PROBLEM FORMULATION}

Fig. 1 shows a general framework of networked control systems. The physical process to be controlled is a linear continuous-time system. The system state is sampled periodically. Let $\left\{t_{k}=k T_{s}: k \in \mathbb{Z}+\right\}$ be the sampling instants, where $T_{s}>0$ is the sampling period. The sampler samples the value of the system state $x\left(t_{k}\right)$ at time $t_{k}$, and transmits the system state packet into the network during the interval $t_{k} \leq t<t_{k+1}$. The networks are unreliable and problem prone. Hence time delays or even packet losses may occur. However, a certain level of communication performance is maintained. That is, the time delays and the number of the consecutive lost packets are upper bounded. The networked controller is time-invariant. It is not necessary to be synchronized with either the sampler or the $\mathrm{ZOH}$, and independent of the time delays and packet losses. The controller simply starts a new computation once it has received a system state packet from the network, and transmits a control input packet into the network after it has completed the computation. The control input package has the same time stamp as the system state package. Because of the time-varying nature of the transmission time delays, control input packets may arrive at the $\mathrm{ZOH}$ with a different temporal order than that with which the corresponding system state packets were transmitted from the sampler. Therefore, the $\mathrm{ZOH}$ is configured to accept the arrived packet only if the time stamp of it is greater than that of the packet that the $\mathrm{ZOH}$ currently stores. Moreover, the $\mathrm{ZOH}$ is synchronized with the sampler by a clock (synchronization here means that the $\mathrm{ZOH}$ adjusts its output only on the 


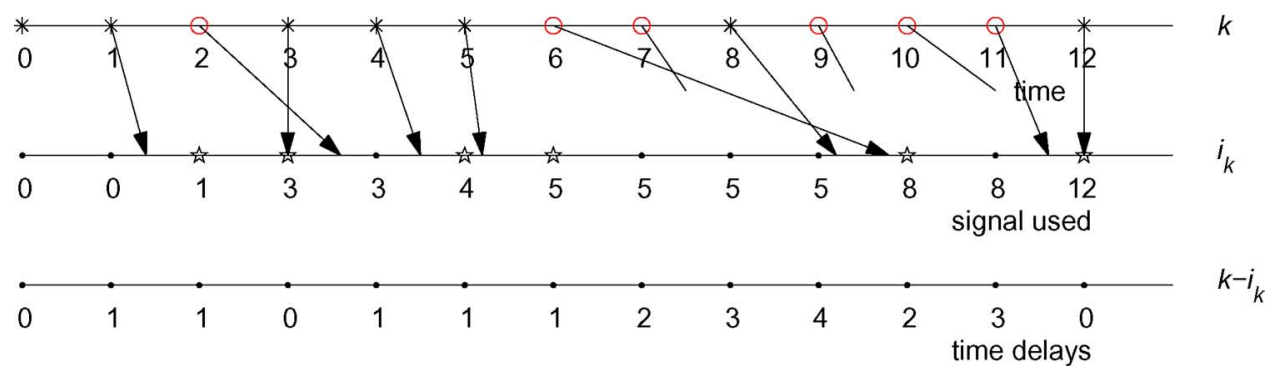

Fig. 2. An example of packets transmitted, lost and used.

sampling instants). The mechanism of the $\mathrm{ZOH}$ can be concisely described as follows.

Logic ZOH: Given $u(0)$, let $i_{0}=0$ and $k=0$.

1 At sampling instant $t_{k}, \mathrm{ZOH}$ changes its output to $u(t)=u\left(i_{k} T_{s}\right)$ for $t_{k} \leq t<t_{k+1}$. Let $i_{k+1}=i_{k}$.

2 During $t_{k}<t \leq t_{k+1}$, if a packet $u\left(j T_{s}\right)$ arrives and $j>i_{k+1}$, then ZOH stores $u\left(j T_{s}\right)$ and lets $i_{k+1}=j$.

3 Repeat Step 2 until $t$ reaches the next sampling instant $t_{k+1}$. Let $k=k+1$ and go to Step 1 .

Remark 1: In the above description, $u\left(i_{k} T_{s}\right)$ is the newest control information available to the logic $\mathrm{ZOH}$ up to time $t_{k} ; u\left(i_{k+1} T_{s}\right)$ is the newest control information available to $\mathrm{ZOH}$ up to time $t$, where $t_{k}<$ $t \leq t_{k+1} ; u\left(j T_{s}\right)$ is the control information arriving at $\mathrm{ZOH}$ during $t_{k}<t \leq t_{k+1}$. The time instants $i_{k}, i_{k+1}$ and $j$ can be considered as the time stamps of the control input packets. In Step 1, the ZOH updates its output with the newest control information. In Step 2, the ZOH only holds the most up-to-date control information, which will be used in the next sampling period. To complete this task, the $\mathrm{ZOH}$ is supplied with a logic to compare and a memory to store the control input packets. In addition, we have both $i_{k} \leq k$ and $i_{k} \leq i_{k+1} \leq k+1$.

Let us define the input delay $\tau(k) \triangleq k-i_{k}$ for $t_{k} \leq t<t_{k+1}$. Then the input $u(t)$ in Step 1 of Logic ZOH can be represented by $u(t)=u\left((k-\tau(k)) T_{s}\right)$. As $i_{k+1} \geq i_{k}$, we have $\tau(k+1) \leq \tau(k)+1$. On the other hand, the characteristic of the networks ensures that $\tau(k)$ has an upper bound, that is, $0 \leq \tau(k) \leq \tau_{\max }$, which means that at least one new packet is accepted and used by ZOH every $\tau_{\max } T_{s}$.

The time delays and packet losses of the network transmissions are merged into the input delay $\tau(k)$. The sequence of the input delay values provides the information needed to identify the transmission time delays and packet losses. We already have $\tau(k+1) \leq \tau(k)+1$. Then there are two cases. Case 1: $\tau(k+1)=\tau(k)+1$, this is equivalent to that no new packet arrives during $t_{k}<t \leq t_{k+1}$. The ZOH continues to apply the same control input, so the input delay is increased by one. Case 2: $\tau(k+1) \leq \tau(k)$, this case is the same as that a new packet of delay $\tau(k+1)$ is accepted by the $\mathrm{ZOH}$ up to time $t_{k+1}$. This packet will be used in the next sampling period, and $\tau(k)-\tau(k+1)$ consecutive packets prior to this packet have been lost during the transmission. Fig. 2 illustrates these cases.

Based upon the analysis above, the continuity of the process in Fig. 1, together with the sampler and the logic $\mathrm{ZOH}$, can be discretized as a linear discrete-time system with input delays:

$$
x(k+1)=A x(k)+B u(k-\tau(k))
$$

where $k \in \mathbb{Z}_{+}$is the time step, $x(k) \in \mathbb{R}^{n}$ and $u(k) \in \mathbb{R}^{m}$ are the system state and the control input, respectively. $\tau(k)$ is the input delay satisfying $0 \leq \tau(k) \leq \tau_{\max }$ with $\tau_{\max }>0$ and $\tau(k+1) \leq$ $\tau(k)+1$. The initial system state is $x_{0} \triangleq x(0) . A$ and $B$ are two constant matrices of appropriate dimensions. Here we have omitted the sampling period $T_{s}$ for simplicity.

In this technical note, we are interested in designing a state-feedback controller:

$$
u=K x
$$

where $K$ is to be designed. The time step $k$ is omitted in (2) to indicate that the controller is event-driven only and is independent of the time delay and packet loss issues of the network transmission. Then the resulting closed-loop system is a time-delay system:

$$
x(k+1)=A x(k)+B K x(k-\tau(k)), \quad k \in \mathbb{Z}_{+}
$$

The objective of this technical note is to design the networked controller (2) such that networked control system (3) is asymptotically stable.

Remark 2: Our proposed framework is similar to those in [17]-[20]. In [17], the $\mathrm{ZOH}$ is event-driven only, and the newest control input takes effect immediately. In [18], the input delay $\tau(k)$ is further divided into two parts: one from sampler to controller and the other from controller to ZOH. In [19], [20], the ZOH has no logical decision and always uses the latest arrived control information. Specifically, $\tau(k+1) \leq \tau(k)+1$ is not guaranteed.

Remark 3: It is worth mentioning that the time delay and the packet loss of the network transmissions manifest themselves in the input delays of model (1). The values of the time delay and the packet loss can be derived from the values of the input delay and vice versa.

\section{MAIN RESULTS}

In this section, we present a sufficient condition for the stability analysis and two sufficient conditions for the synthesis of networked control systems. The following theorem provides us the stability condition of networked control system (3), and plays an essential role in the controller design.

Theorem 1: NCS (3) is asymptotically stable if there exist matrices $P \in \mathbb{S}^{+}, Z \in \mathbb{S}^{+}, T_{1} \in \mathbb{R}^{n \times n}$ and $T_{2} \in \mathbb{R}^{n \times n}$ satisfying

$$
\left[\begin{array}{ccc}
\Phi_{11} & \Phi_{12} & T_{1} \\
\Phi_{12}^{T} & \Phi_{22} & T_{2} \\
T_{1}^{T} & T_{2}^{T} & -\frac{1}{\tau_{\max } Z}
\end{array}\right]<0
$$


where

$$
\begin{aligned}
& \Phi_{11}=A^{T} P A-P+\tau_{\max }\left(A^{T}-I\right) Z(A-I)+T_{1}+T_{1}^{T} \\
& \Phi_{12}=A^{T} P B K+\tau_{\max }\left(A^{T}-I\right) Z B K-T_{1}+T_{2}^{T} \\
& \Phi_{22}=K^{T} B^{T} P B K+\tau_{\max } K^{T} B^{T} Z B K-T_{2}-T_{2}^{T}
\end{aligned}
$$

Proof: To facilitate the proof, we define the following symbols:

$$
\begin{aligned}
& x_{k} \triangleq\left[\begin{array}{llll}
x^{T}(k) & x^{T}(k-1) & \cdots & x^{T}\left(k-\tau_{\max }\right)
\end{array}\right]^{T} \\
& \xi(k) \triangleq\left[x^{T}(k) \quad x^{T}(k-\tau(k))\right]^{T} \\
& \zeta(k) \triangleq x(k+1)-x(k)
\end{aligned}
$$

and have

$$
\begin{aligned}
\zeta(k) & =(A-I) x(k)+B K x(k-\tau(k)) \\
\sum_{h=k-\tau(k)}^{k-1} \zeta(h) & =x(k)-x(k-\tau(k))
\end{aligned}
$$

Now take the Lyapunov functional as

$$
V\left(x_{k}, k\right) \triangleq V_{1}\left(x_{k}, k\right)+V_{2}\left(x_{k}, k\right)+V_{3}\left(x_{k}, k\right)
$$

where

$$
\begin{aligned}
& V_{1}\left(x_{k}, k\right)=x^{T}(k) \operatorname{Px}(k) \\
& V_{2}\left(x_{k}, k\right)=\sum_{l=k-\tau(k)}^{k-1} x^{T}(l) Q x(l) \\
& V_{3}\left(x_{k}, k\right)=\sum_{l=-\tau_{\max }+1}^{0} \sum_{h=k-1+l}^{k-1} \zeta^{T}(h) Z \zeta(h)
\end{aligned}
$$

Then

$$
\begin{aligned}
& V_{1}\left(x_{k+1}, k+1\right)-V_{1}\left(x_{k}, k\right) \\
& \quad=x^{T}(k+1) P x(k+1)-x^{T}(k) P x(k) \\
& \quad=\xi^{T}(k)\left[\begin{array}{cc}
A^{T} P A-P & A^{T} P B K \\
K^{T} B^{T} P A & K^{T} B^{T} P B K
\end{array}\right] \xi(k)
\end{aligned}
$$

Noticing that $k-\tau(k) \leq k+1-\tau(k+1)$, we have

$$
\begin{aligned}
& V_{2}\left(x_{k+1}, k+1\right)-V_{2}\left(x_{k}, k\right) \\
& =x^{T}(k) Q x(k)+\sum_{l=k+1-\tau(k+1)}^{k-1} x^{T}(l) Q x(l) \\
& \quad-\sum_{l=k-\tau(k)}^{k-1} x^{T}(l) Q x(l) \\
& \leq x^{T}(k) Q x(k)
\end{aligned}
$$

Finally

$$
\begin{aligned}
& V_{3}\left(x_{k+1}, k+1\right)-V_{3}\left(x_{k}, k\right) \\
& =\sum_{l=-\tau_{\max }+1}^{0}\left[\sum_{h=k+l}^{k} \zeta^{T}(h) Z \zeta(h)-\sum_{h=k-1+l}^{k-1} \zeta^{T}(h) Z \zeta(h)\right] \\
& =\sum_{l=-\tau_{\max }+1}^{0}\left[\zeta^{T}(k) Z \zeta(k)-\zeta^{T}(k-1+l) Z \zeta(k-1+l)\right] \\
& =\tau_{\max } \zeta^{T}(k) Z \zeta(k)-\sum_{l=k-\tau_{\max }}^{k-1} \zeta^{T}(l) Z \zeta(l)
\end{aligned}
$$

Noticing that for $Z>0$ and any matrix $T=\left[\begin{array}{l}T_{1} \\ T_{2}\end{array}\right] \in \mathbb{R}^{2 n \times n}$, we have

$$
\left[\begin{array}{cc}
T Z^{-1} T^{T} & T \\
T^{T} & Z
\end{array}\right] \geq 0
$$

Hence

$$
\begin{aligned}
0 \leq & \sum_{l=k-\tau(k)}^{k-1}\left[\begin{array}{c}
\xi(k) \\
\zeta(l)
\end{array}\right]^{T}\left[\begin{array}{cc}
T Z^{-1} T^{T} & T \\
T^{T} & Z
\end{array}\right]\left[\begin{array}{l}
\xi(k) \\
\zeta(l)
\end{array}\right] \\
= & \tau(k) \xi^{T}(k) T Z^{-1} T^{T} \xi(k)+2 \xi^{T}(k) T \sum_{l=k-\tau(k)}^{k-1} \zeta(l) \\
& +\sum_{l=k-\tau(k)}^{k-1} \zeta^{T}(l) Z \zeta(l) \\
\leq & \tau_{\max } \xi^{T}(k) T Z^{-1} T^{T} \xi(k) \\
& +2 \xi^{T}(k) T[x(k)-x(k-\tau(k))]+\sum_{l=k-\tau_{\max }}^{k-1} \zeta^{T}(l) Z \zeta(l)
\end{aligned}
$$$$
\triangleq \Gamma
$$

Thus

$$
\begin{aligned}
& V_{3}\left(x_{k+1}, k+1\right)-V_{3}\left(x_{k}, k\right) \\
& \leq V_{3}\left(x_{k+1}, k+1\right)-V_{3}\left(x_{k}, k\right)+\Gamma \\
& \leq \xi^{T}(k)\left(\tau_{\max }\left[\begin{array}{l}
T_{1} \\
T_{2}
\end{array}\right] Z^{-1}\left[\begin{array}{ll}
T_{1}^{T} & T_{2}^{T}
\end{array}\right]\right. \\
& +\tau_{\max }\left[\begin{array}{cc}
\left(A^{T}-I\right) Z(A-I) & \left(A^{T}-I\right) Z B K \\
K^{T} B^{T} Z(A-I) & K^{T} B^{T} Z B K
\end{array}\right] \\
& \left.+\left[\begin{array}{cc}
T_{1}+T_{1}^{T} & -T_{1}+T_{2}^{T} \\
-T_{1}^{T}+T_{2} & -T_{2}-T_{2}^{T}
\end{array}\right]\right) \xi(k)
\end{aligned}
$$

Therefore, for any $\xi(k) \neq 0$, we have

$$
\begin{aligned}
& V\left(x_{k+1}, k+1\right)-V\left(x_{k}, k\right) \\
& \leq \xi^{T}(k)\left(\left[\begin{array}{cc}
\Phi_{11}+Q & \Phi_{12} \\
\Phi_{12}^{T} & \Phi_{22}
\end{array}\right]\right. \\
& \left.\quad+\tau_{\max }\left[\begin{array}{l}
T_{1} \\
T_{2}
\end{array}\right] Z^{-1}\left[\begin{array}{ll}
T_{1}^{T} & T_{2}^{T}
\end{array}\right]\right) \xi(k) \\
& \quad<0
\end{aligned}
$$

if LMI (4) holds and $Q$ is set to be small enough.

Remark 4: The use of the logic ZOH ensures that $k-\tau(k) \leq k+1-$ $\tau(k+1)$, which further guarantees that $\sum_{l=k+1-\tau(k+1)}^{k-1} x^{T}(l) Q x(l)-$ $\sum_{l=k-\tau(k)}^{k-1} x^{T}(l) Q x(l) \leq 0$. This inequality however does not always hold for a general time-delay system, where an additional term such as $\sum_{l=-\tau_{\max }+1}^{0} \sum_{h=k-1+l}^{k-1} x^{T}(h) Q x(h)$ is often introduced in $V_{2}$ to cancel the positive term $\sum_{l=k+1-\tau(k+1)}^{k-1} x^{T}(l) Q x(l)$ [22]. In our case, there is no need to introduce such additional term in $V_{2}$, and it is further shown in the proof that $V_{2}$ is redundant since $Q>0$ can be set sufficiently small in a positive definite sense.

Based upon Theorem 1, we are ready to present two procedures for the controller design. They can be combined together to balance the computational complexity and conservatism of the conditions.

Lemma 1: Consider system (1), there exists a networked controller (2) such that NCS (3) is asymptotically stable if there exist matrices 
$P \in \mathbb{S}^{+}, X \in \mathbb{S}^{+}, Z \in \mathbb{S}^{+}, W \in \mathbb{S}^{+}, T_{1} \in \mathbb{R}^{n \times n}, T_{2} \in \mathbb{R}^{n \times n}$ and $K \in \mathbb{R}^{m \times n}$ satisfying LMI

$$
\left[\begin{array}{ccccc}
\Psi_{1} & \bullet & \bullet & \bullet & \bullet \\
-T_{1}^{T}+T_{2} & -T_{2}-T_{2}^{T} & \bullet & \bullet & \bullet \\
T_{1}^{T} & T_{2}^{T} & -\frac{1}{\tau_{\max }} Z & \bullet & \bullet \\
A-I & B K & 0 & -\frac{1}{\tau_{\max }} W & 0 \\
A & B K & 0 & 0 & -X
\end{array}\right]<0
$$

with equality constraints

$$
Z W=I, \quad P X=I
$$

where $\Psi_{1}=-P+T_{1}+T_{1}^{T}$.

Proof: Note that (4) is equivalent to

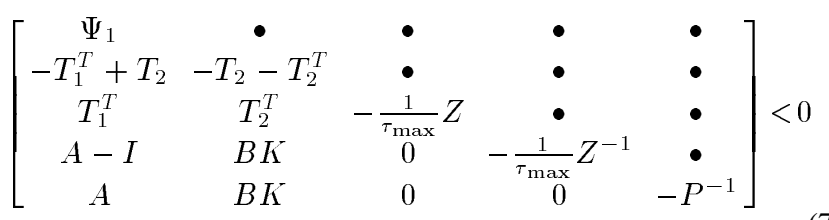

This inequality is further equivalent to (5) and (6) if we define $W \triangleq$ $Z^{-1}$ and $X \triangleq P^{-1}$.

Although the cone complementarity linearization type algorithms [23], [24] are efficient to solve such problems, in most cases, we prefer LMI conditions since they can be solved more directly. Next we provide an $\mathrm{LMI}$ condition.

Lemma 2: Consider system (1), there exists a networked controller (2) such that NCS (3) is asymptotically stable if there exist matrices $X \in \mathbb{S}^{+}, W \in \mathbb{S}^{+}, \bar{T}_{1} \in \mathbb{R}^{n \times n}, \bar{T}_{2} \in \mathbb{R}^{n \times n}$ and $Y \in \mathbb{R}^{m \times n}$ such that LMI

$$
\left[\begin{array}{ccccc}
\Psi_{2} & \bullet & \bullet & \bullet & \bullet \\
-\bar{T}_{1}^{T}+\bar{T}_{2} & -\bar{T}_{2}-\bar{T}_{2}^{T} & \bullet & \bullet & \bullet \\
\bar{T}_{1}^{T} & \bar{T}_{2}^{T} & \Psi_{3} & \bullet & \bullet \\
(A-I) X & B Y & 0 & -\frac{1}{\tau_{\max }} W & \bullet \\
A X & B Y & 0 & 0 & -X
\end{array}\right]<0
$$

holds for some scalar $\alpha>0$, where $\Psi_{2}=-X+\bar{T}_{1}+\bar{T}_{1}^{T}$ and $\Psi_{3}=-\left(1 / \tau_{\max }\right)\left(2 \alpha X-\alpha^{2} W\right)$. In this case, the controller is given by $K=Y X^{-1}$.

Proof: Pre- and post-multiplying (7) by $\operatorname{diag}\left(P^{-1}, P^{-1}, P^{-1}, I, I\right)$, and defining $X \triangleq P^{-1}$, $Y \triangleq K P^{-1}, W \triangleq Z^{-1}, \bar{T}_{1} \triangleq P^{-1} T_{1} P^{-1}, \bar{T}_{2} \triangleq P^{-1} T_{2} P^{-1}$, we have

$$
\left[\begin{array}{ccccc}
\Psi_{2} & \bullet & \bullet & \bullet & \bullet \\
-\bar{T}_{1}^{T}+\bar{T}_{2} & -\bar{T}_{2}-\bar{T}_{2}^{T} & \bullet & \bullet & \bullet \\
\bar{T}_{1}^{T} & \bar{T}_{2}^{T} & \Psi_{4} & \bullet & \bullet \\
(A-I) X & B Y & 0 & -\frac{1}{\tau_{\max }} W & \bullet \\
A X & B Y & 0 & 0 & -X
\end{array}\right]<0
$$

where $\Psi_{4}=-\left(1 / \tau_{\max }\right) X W^{-1} X$. Note that $(X-$ $\alpha W)(\alpha W)^{-1}(X-\alpha W) \geq 0$ implies $2 \alpha X-\alpha^{2} W \leq X W^{-1} X$, that is, $\Psi_{4} \leq \Psi_{3}$. Therefore, the solvability of LMI (8) for some given scalar $\alpha>0$ guarantees the solvability of inequality (9).

Remark 5: Lemma 1 and Lemma 2 can be combined to design the controller. First one should try to solve (8) for some scalars $\alpha>0$. If a solution cannot be found, then try to solve (5) and (6).

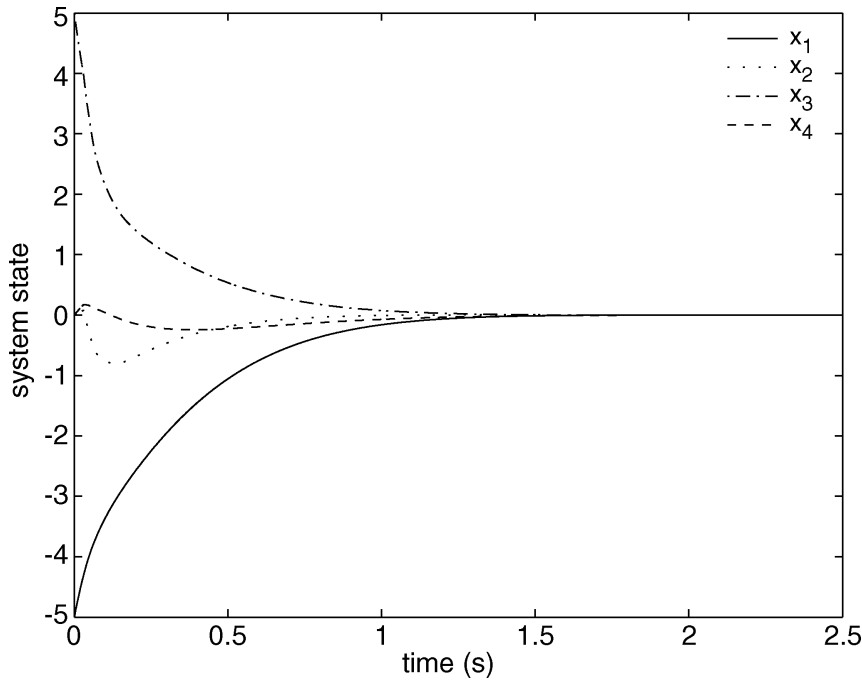

Fig. 3. Initial condition response of the closed-loop system.

\section{EXAMPLES}

In this section, we illustrate the developed theory via several numerical examples and simulations. The continuous-time processes are taken from the recently published papers. Here, we first discretize them to their corresponding discrete-time versions, then apply the controller design methods in this technical note to construct the networked controllers, and finally we conduct simulations based upon the framework in Fig. 1 in Section II. For the transmission characteristic of the networks, $1 \leq \tau(k) \leq 5$ is assumed. The initial control input is chosen to be zero.

Example 1: Consider an unstable batch reactor [1]:

$\dot{x}(t)=\left[\begin{array}{cccc}1.38 & -0.2077 & 6.715 & -5.676 \\ -0.5814 & -4.29 & 0 & 0.675 \\ 1.067 & 4.273 & -6.654 & 5.893 \\ 0.048 & 4.273 & 1.343 & -2.104\end{array}\right] x(t)$
+
+
$+\left[\begin{array}{cc}0 & 0 \\ 5.679 & 0 \\ 1.136 & -3.146 \\ 1.136 & 0\end{array}\right] u(t)$

We discretize the system with $T_{s}=0.005 \mathrm{~s}$ and obtain

$x(k+1)=\left[\begin{array}{cccc}1.0070 & -0.0010 & 0.0330 & -0.0278 \\ -0.0029 & 0.9788 & -0.0000 & 0.0034 \\ 0.0052 & 0.0211 & 0.9675 & 0.0288 \\ 0.0002 & 0.0211 & 0.0066 & 0.9897\end{array}\right] x(k)$

$$
+\left[\begin{array}{cc}
0.0000 & -0.0003 \\
0.0281 & 0.0000 \\
0.0060 & -0.0155 \\
0.0060 & -0.0001
\end{array}\right] u(k)
$$

Based upon Lemma 2 with $\alpha=1$, a networked controller is

$$
u=\left[\begin{array}{llll}
0.9762 & -2.5469 & 0.1292 & -1.8632 \\
4.4068 & -0.3153 & 5.2231 & -1.1676
\end{array}\right] x
$$

The initial condition response of the closed-loop system is plotted in Fig. 3, and Fig. 4 shows the distribution of the transmission time delays and packet losses. The initial system state is given by $x_{0}=\left[\begin{array}{lll}-5 & 0 & 5\end{array}\right]^{T}$. 


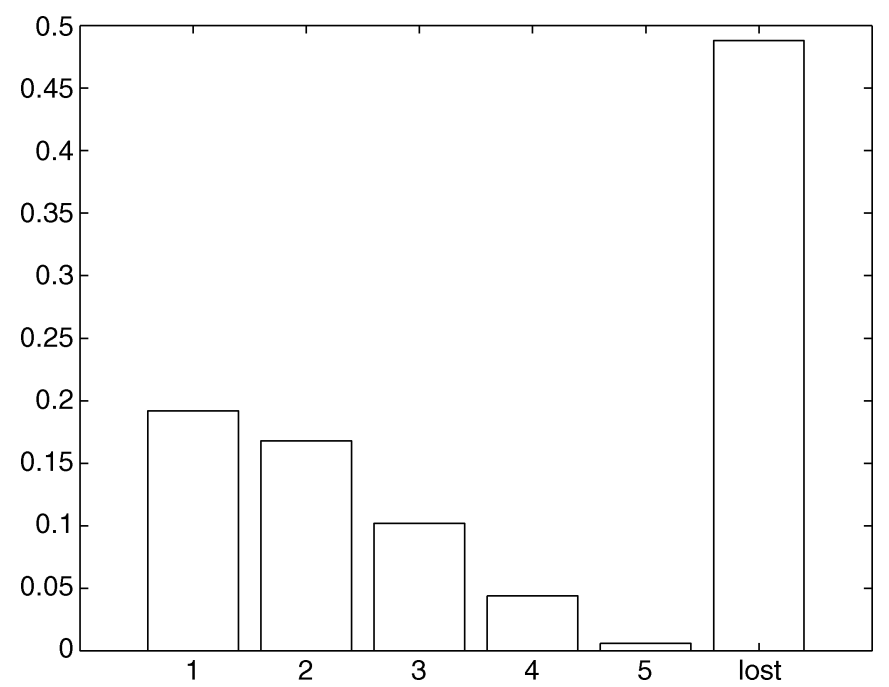

Fig. 4. Distribution of time delays and packet losses.

Example 2: Consider the linearized state-space model of motion about the upward unstable equilibrium position of a pendulum [8]:

$$
\dot{x}(t)=\left[\begin{array}{cccc}
0 & 1 & 0 & 0 \\
63.25 & 0 & 0 & 0 \\
0 & 0 & 0 & 1 \\
-33.31 & 0 & 0 & 0
\end{array}\right] x(t)+\left[\begin{array}{c}
0 \\
-520.72 \\
0 \\
804.13
\end{array}\right] u(t)
$$

The discretized system at $T_{s}=0.005 \mathrm{~s}$ is

$$
\begin{aligned}
x(k+1)= & {\left[\begin{array}{cccc}
1.0008 & 0.0050 & 0.0000 & 0.0000 \\
0.3163 & 1.0008 & 0.0000 & 0.0000 \\
-0.0004 & -0.0000 & 1.0000 & 0.0050 \\
-0.1666 & -0.0004 & 0.0000 & 1.0000
\end{array}\right] x(k) } \\
& +\left[\begin{array}{c}
-0.0065 \\
-2.6043 \\
0.0101 \\
4.0210
\end{array}\right] u(k)
\end{aligned}
$$

Lemma 2 with $\alpha=1$ provides us with a networked controller

$$
u=\left[\begin{array}{llll}
0.3662 & 0.0539 & 0.0034 & 0.0082
\end{array}\right] x
$$

A simulation is given in Fig. 5 and Fig. 6, and the initial system state is $x_{0}=\left[\begin{array}{llll}-5 & 0 & 5 & 0\end{array}\right]^{T}$.

Example 3: Consider the unstable dynamic system in [17]

$$
\dot{x}(t)=\left[\begin{array}{ccc}
-1 & 0 & -0.5 \\
1 & -0.5 & 0 \\
0 & 0 & 0.5
\end{array}\right] x(t)+\left[\begin{array}{l}
0 \\
0 \\
1
\end{array}\right] u(t)
$$

when the sampling period is chosen as $T_{s}=0.2 \mathrm{~s}$, the system is discretized as

$$
\begin{aligned}
x(k+1)=\left[\begin{array}{ccc}
0.8187 & 0.0000 & -0.0955 \\
0.1722 & 0.9048 & -0.0094 \\
0.0000 & 0.0000 & 1.1052
\end{array}\right] x(k) \\
+\left[\begin{array}{c}
-0.0097 \\
-0.0006 \\
0.2103
\end{array}\right] u(k) .
\end{aligned}
$$

Lemma 1 gives us a networked controller

$$
u=\left[\begin{array}{lll}
0.0599 & 0.0261 & -0.8385
\end{array}\right] x
$$

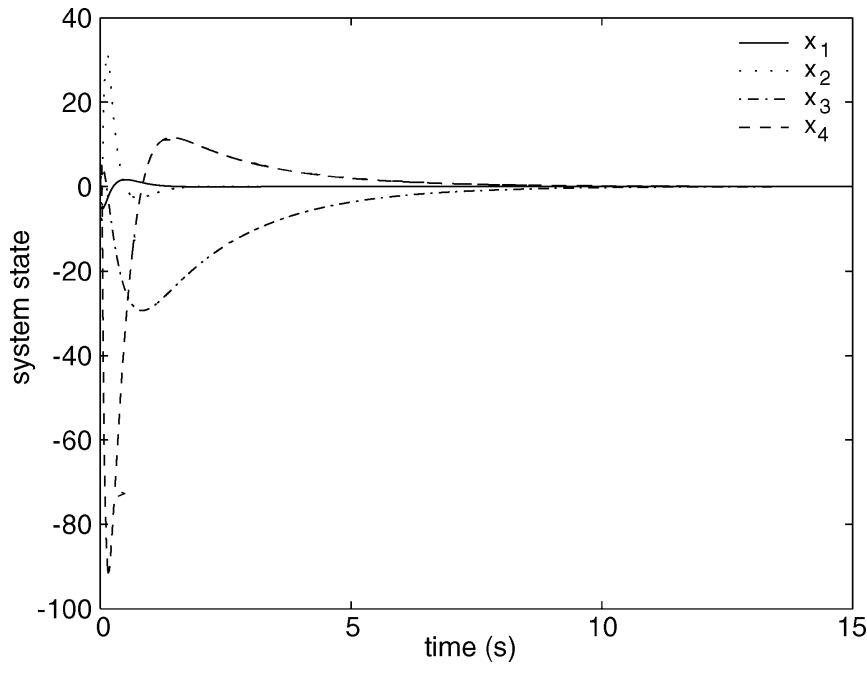

Fig. 5. Initial condition response of the closed-loop system.

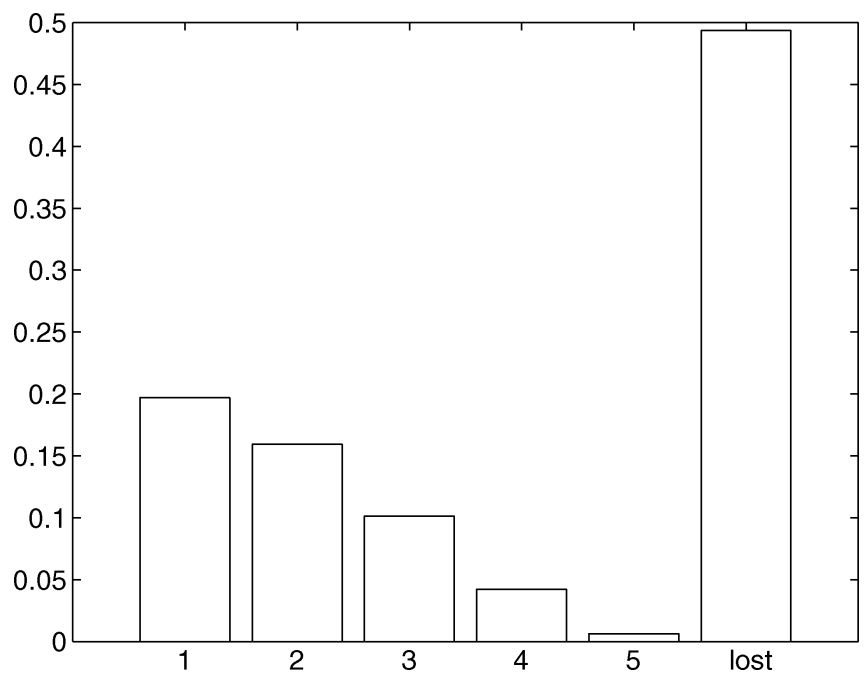

Fig. 6. Distribution of time delays and packet losses.

A simulation is given in Figs. 7 and 8, and the initial system state is $x_{0}=\left[\begin{array}{lll}-5 & 0 & 5\end{array}\right]^{T}$.

In the simulations, $\tau(0)$ is set to be zero and $\tau(k+1)$ is first generated uniformly distributed over $\{1, \ldots, 5\}$, then compared to $\tau(k)+1$. If $\tau(k+1) \geq \tau(k)+1$, we let $\tau(k+1)=\tau(k)+1$ to force the $\mathrm{ZOH}$ to use the newest control information. This is also the reason why the percentages of larger delays are smaller. The transmission time delays and packet losses are calculated from the values of $\tau(k)$.

Example 4: Consider the unstable discrete-time system in [19]:

$$
x(k+1)=\left[\begin{array}{cc}
0.8 & 0.002 \\
0 & 1
\end{array}\right] x(k)+\left[\begin{array}{c}
0 \\
0.3
\end{array}\right] u(k)
$$

The optimization procedure in [19] gives the maximum upper bound of the input delay $\tau(k)$ as 3 (the reported value in [19] was 3.6239, but integral value is considered here). However, applying Lemma 2 with $\alpha=0.5$ we can obtain a stabilizing controller

$$
u=[0.0005-0.1304] x
$$

for $\tau_{\max }=15$. Therefore, the results in this technical note are much less conservative than those in [19]. 


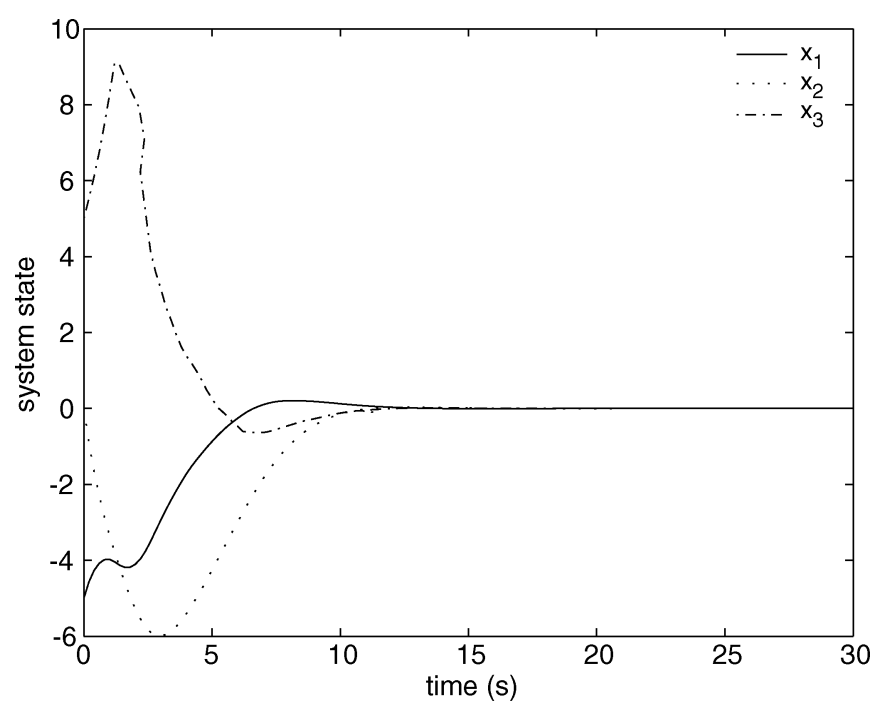

Fig. 7. Initial condition response of the closed-loop system.

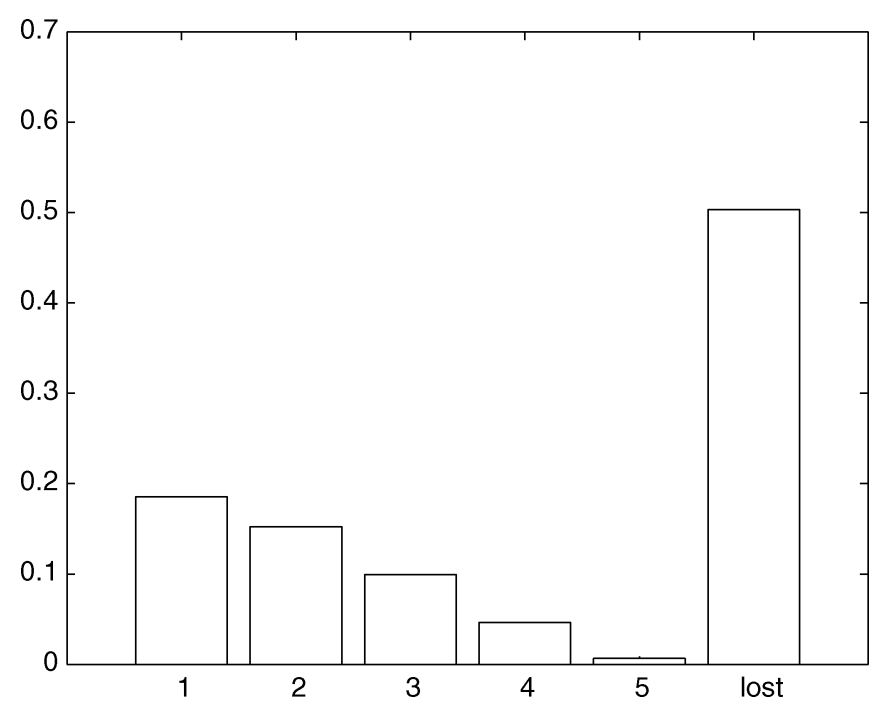

Fig. 8. Distribution of time delays and packet losses.

\section{CONCLUSION}

The stabilization problem of networked control systems has been studied in the technical note. We have first discretized the continuoustime system to a discrete-time system with input delays, then addressed several sufficient conditions for the stability and stabilization of the discretized networked control systems based upon the Lyapunov theory. Finally, the developed theory has been illustrated by the numerical examples and simulations.

One assumption of the theory developed in this technical note is that the components of the system state are sampled simultaneously at the sampling instants. A possible relaxation of this is to allow the sampler sampling the system state within the sampling intervals. Another relaxation approach is to use multiple sensors to sample the system state. In those cases, we may treat the difference between the sampled values and the values of the system state at the sampling instants as uncertainty, then the techniques from robust control may be borrowed.

Another assumption is that the sensor and the actuator are synchronized at sampling instants. A possible way to remove this requirement is to use multiple sensors/actuators and to synchronize them at different sampling instants. In this case, the closed-loop networked control system becomes a discrete-time system with multiple input delays, and co-design techniques might be used. That is, the communication network is first designed to satisfy certain performance and then the controller is designed.

\section{REFERENCES}

[1] G. C. Walsh and H. Ye, "Scheduling of networked control systems," IEEE Control Syst. Mag., vol. 21, no. 1, pp. 57-65, 2001.

[2] Y. Tipsuwan and M.-Y. Chow, "Control methodologies in networked control systems," Control Eng. Practice, vol. 11, no. 10, pp. 1099-1111, 2003.

[3] T. C. Yang, "Networked control system: A brief survey," Proc. Inst. Elect. Eng., vol. 153, no. 4, pp. 403-412, 2006.

[4] D. Hristu-Varsakelis, "Feedback control with communication constraints," in Handbook of Networked and Embedded Control Systems. Boston, MA: Birkhauser, 2005, pp. 575-599.

[5] J. Nilsson, B. Bernhardsson, and B. Wittenmark, "Stochastic analysis and control of real-time systems with random time delays," Automatica, vol. 34, no. 1, pp. 57-64, 1998.

[6] W. Zhang, M. S. Branicky, and S. M. Phillips, "Stability of networked control systems," IEEE Control Syst. Mag., vol. 21, no. 1, pp. 84-99, 2001.

[7] D. S. Kim, Y. S. Lee, W. H. Kwon, and H. S. Park, "Maximum allowable delay bounds of networked control systems," Control Eng. Practice, vol. 11, no. 11, pp. 1301-1313, 2003.

[8] N. J. Ploplys, P. A. Kawka, and A. G. Alleyne, "Closed-loop control over wireless networks," IEEE Control Syst. Mag., vol. 24, no. 3, pp. 58-71, 2004.

[9] Z. Wang, D. W. C. Ho, and X. Liu, "Variance-constrained filtering for uncertain stochastic systems with missing measurements," IEEE Trans. Automat. Control, vol. 48, no. 7, pp. 1254-1258, Jul. 2003.

[10] D. K. Kim, P. Park, and J. W. Ko, "Output-feedback $H_{\infty}$ control of systems over communication networks using a deterministic switching system approach," Automatica, vol. 40, no. 7, pp. 1205-1212, 2004.

[11] P. Seiler and R. Sengupta, "An $H_{\infty}$ approach to networked control," IEEE Trans. Automat. Control, vol. 50, no. 3, pp. 356-364, Mar. 2005.

[12] F. Yang, Z. Wang, Y. S. Hung, and M. Gani, " $H_{\infty}$ control for networked systems with random communication delays," IEEE Trans. Automat. Control, vol. 51, no. 3, pp. 511-518, Mar. 2006.

[13] Y.-J. Pan, H. J. Marquez, and T. Chen, "Stabilization of remote control systems with unknown time varying delays by LMI techniques," Int. J. Control, vol. 79, no. 7, pp. 752-763, 2006.

[14] J. Lam, H. Gao, and C. Wang, "Stability analysis for continuous systems with two additive time-varying delay components," Syst. Control Lett., vol. 56, no. 1, pp. 16-24, 2007.

[15] H. Gao, J. Lam, T. Chen, and C. Wang, "Feedback control with signal transmission after-effects," Int. J. Robust Nonlin. Control, vol. 18, no. 3, pp. 351-363, 2008.

[16] H. Gao, T. Chen, and J. Lam, "A new delay system approach to network-based control," Automatica, vol. 44, no. 1, pp. 39-52, 2008.

[17] D. Yue, Q.-L. Han, and J. Lam, "Network-based robust $H_{\infty}$ control of systems with uncertainty," Automatica, vol. 41, no. 6, pp. 999-1007, 2005.

[18] L. Zhang, Y. Shi, T. Chen, and B. Huang, "A new method for stabilization of networked control systems with random delays," IEEE Trans. Automat. Control, vol. 50, no. 8, pp. 1177-1181, Aug. 2005.

[19] M. Yu, L. Wang, T. Chu, and F. Hao, "An LMI approach to networked control systems with data packet dropout and transmission delays," in Proc. Conf. Decision Control, 2004, pp. 3545-3550.

[20] M. Yu, L. Wang, and . Chu, "An LMI approach to robust stabilization of networked control systems," in Proc. IFAC World Congress, 2005, [CD ROM]

[21] L. Zhang and D. Hristu-Varsakelis, "Communication and control co-design for networked control systems," Automatica, vol. 42, no. 6 , pp. 953-958, 2006

[22] S. Xu, J. Lam, and T. Chen, "Robust $H_{\infty}$ control for uncertain discrete stochastic time-delay systems," Syst. Control Lett., vol. 51, no. 3-4, pp. 203-215, 2004.

[23] L. El Ghaoui, F. Oustry, and M. A. Rami, "A cone complementarity linearization algorithm for static output-feedback and related problems," IEEE Trans. Automat. Control, vol. 42, no. 8, pp. 1171-1176, Aug. 1997.

[24] F. Leibfritz, "An LMI-based algorithm for designing suboptimal static $H_{2} / H_{\infty}$ output feedback controllers," SIAM J. Control Optim., vol. 39 , no. 6 , pp. 1711-1735, 2001. 